\title{
Process for Selecting System Level Assessments for Human System Technologies
}

\author{
James Watts, John Park \\ Jacobs Sverdrup Engineering Service Group
}

Copyright $\odot 2005$ SAE International

\begin{abstract}
The integration of many life support systems necessary to construct a stable habitat is difficult. The correct identification of the appropriate technologies and corresponding interfaces is an exhaustive process. Once technologies are selected secondary issues such as mechanical and electrical interfaces must be addressed. The required analytical and testing work must be approached in a piecewise fashion to achieve timely results. A repeatable process has been developed to identify and prioritize system level assessments and testing needs. This Assessment Selection Process has been defined to assess cross cutting integration issues on topics at the system or component levels. Assessments are used to identify risks, encourage future actions to mitigate risks, or spur further studies.
\end{abstract}

\section{INTRODUCTION}

Processes have been developed to accomplish many system engineering functions. These processes are mainly used for some initial exercise. Engineers continue to develop such schemes to document progress and to plan for future efforts. Some authors have commented about the relative ineffectiveness of some system engineering processes, at least when used as the sole method of evaluation (Jones, 2005).

Programmatic system engineering tools have been developed to help identify and prioritize systems level studies, requiring the attention of the engineering team. These studies are used to resolve design intent, identify overlooked issues, and plan future budgetary allocations.

While the goals of such processes are specific to any particular industry or discipline, the fundamental nature of a selection scheme is to promote uniformity, minimize additional investment, and maximize benefit. These objectives are independent industry or discipline.

A generic selection process has been outlined. An example of a space habitation implementation developed by the AH\&I (Architecture, Habitation and Integration) team has will be presented with emphasis given to Integrated Project Team (IPT) member selection, a preprocessing filter, and the selection of an assessment prioritization scheme.

\section{DEVELOPMENT OF SELECTION PROCESS}

\section{A GENERIC SELECTION PROCESS}

The authors propose that any selection process should adhere to the following guidelines:

- The selection process must be documented and repeatable.

- The selection process can be used at any system, or project level deemed appropriate to investigate.

- The process must allow for management buy-in preventing unsanctioned investment.

- The selection process must be implemented within a relevant time frame.

- The subprocesses used within the selection process must be available to the IPT.

- The IPT must have the latitude to resource preferred selection tools. Some companies have a required selection standard, which can be used. If no standard is required, the IPT should have the latitude to determine what tools are defendable for use in selection.

- The selected assessment results should be presented as a prioritized recommendation based upon a ranking process.

The resulting generic framework was developed as a proposal to address the aforementioned guidelines. 
(Figure 1) The individual subprocesses and documents will now be shown.

\section{Initial Recommendations List}

The initial recommendation list is an acknowledgment of existing assessment work or assumptions. This document should incorporate previous assessment recommendations. This list also provides means to incorporate topics, as recommendations, from non-IPT participants.

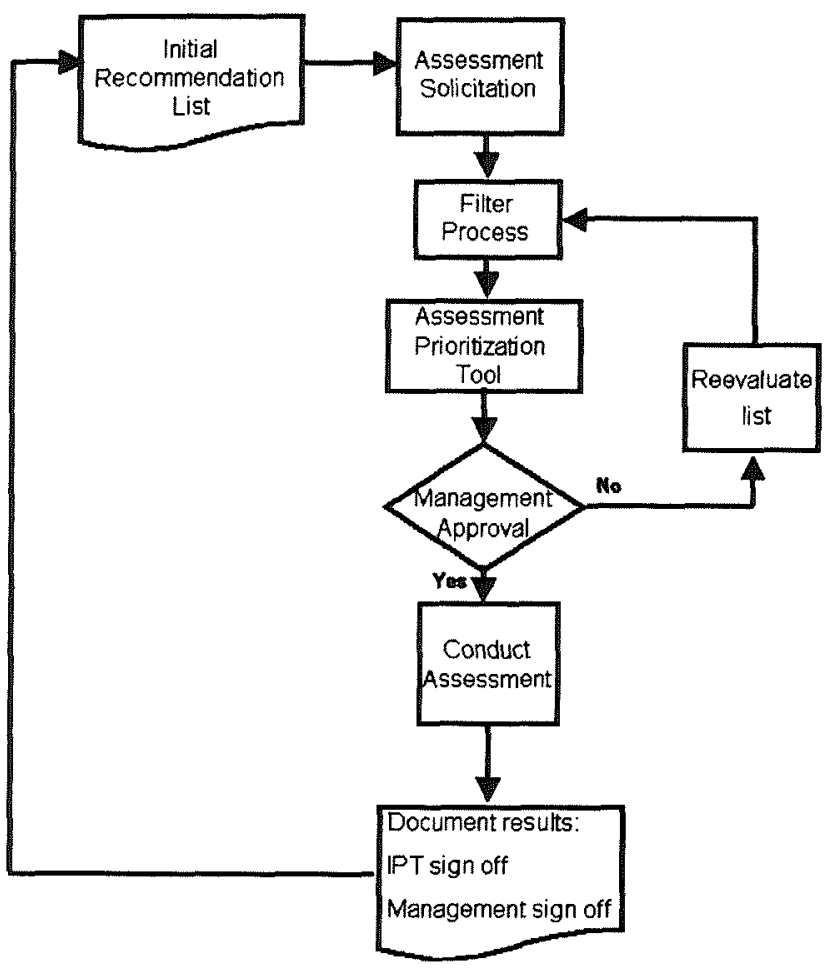

Fig. 1 Generic assessment selection process that satisfies the general guidelines

\section{Assessment Solicitation}

An accepted common manager or coordinator should initiate the assessment selection process. The assessment solicitation subprocess can include three distinct subtasks.

\section{Development of the IPT}

This step includes the selection and recruitment of the IPT members who will perform the Assessment Selection Process. Best practices for group selection based upon teaming dynamics and consensus building should be used. The IPT will contact a selected evaluation community that is to provide information via questionnaires or interviews.

\section{Determination of the Evaluation Community}

A predetermined evaluation community consists of a subset of experts to guide the selection process. The scope of the solicitation must be documented and understood by all parties involved. Individual participation forms or tasking agreements should be used to document the scope of the activity.

\section{Evaluation Community Solicitation}

The evaluation community is to be notified that programmatic information is to be shared for the benefit of the assessment team. Interviews, questionnaires or surveys can be used to gather programmatic and expert based information.

\section{Filter Process}

The filter process is intended to identify duplicate, offtopic, or incomplete assessment subjects. The identified topics may be investigated further by the IPT, or abandoned. This step is intended to furnish the subsequent prioritization subprocess with a relatively small, consistent consideration set. The lists can then be shared and documented as the Assessment Short List. A filter subprocess may be excluded from an abbreviated assessment process.

\section{Assessment Prioritization Tool}

The Prioritization tool is used by the IPT to select programmatically beneficial assessments. The specific tools available for this process vary in complexity and effectiveness. Some assessment strategies are based upon executive determination while others are consensus based. The scheme selected should require the final output to be a quantitative ranking of the assessment topics.

The Analytical Hierarchy Process (AHP) is often used within the NASA R\&D communities with some success. (Saaty 1980) The formality required within the AHP method makes it a desirable option, but the 3 R's method has been suggested for this purpose. (Jones 2005)

\section{Management Approval}

The Management Approval step is used to share the IPT's findings with the accepted management authority. These interactions may result in the acceptance of the IPT's findings and subsequent direction to conduct an assessment, trade study, or test.

Should the management team provide additional instruction, or if the scope of the activity has changed, the IPT may decide to modify the filter and prioritization criteria and reuse the previously collected questionnaire data. This additional step has been included as the Reevaluate List subprocess in figure 1. 


\section{Conduct Assessment}

The IPT may elect to follow a predefined assessment study process. Such processes are usually defined within a given industry's culture, or business practices.

Findings of previous assessment selection loops can be redirected as input into the Recommendations List.

\section{Documents Results}

Assessment results should be documented within a configuration management system or an archive.

\section{HUMAN SUBJECT ASSESSMENT PROCESS DEVELOPMENT}

\section{Modification of the Generic Process}

There is significant benefit from implementing early system engineering principals and conducting evaluation level testing to complex NASA related programs. To properly address questions and help develop proven requirements in habitat systems, will necessitate the use of assessments and trade studies. The AH\&I (Architecture, Habitation and Integration) team was developed to help the JSC and NASA communities to initially explore the risks associated with the proper development of interior habitat environments. AH\&I through an integrated team from JSC has identified, discussed, and collected a list of habitation issues to address via the use of system assessments.

The generic process discussed earlier has been developed further to accommodate the specific needs of NASA's Johnson Space Center (JSC).

Specific implementation of the generic assessment process as related to the NASA JSC AH\&l assessment selection process are discussed in the following example (Figure 2). Detail will be given to the development of the IPT member selection, the Filter Subprocess, and an implementation of the AHP based assessment prioritization scheme.

\section{DRAFT}

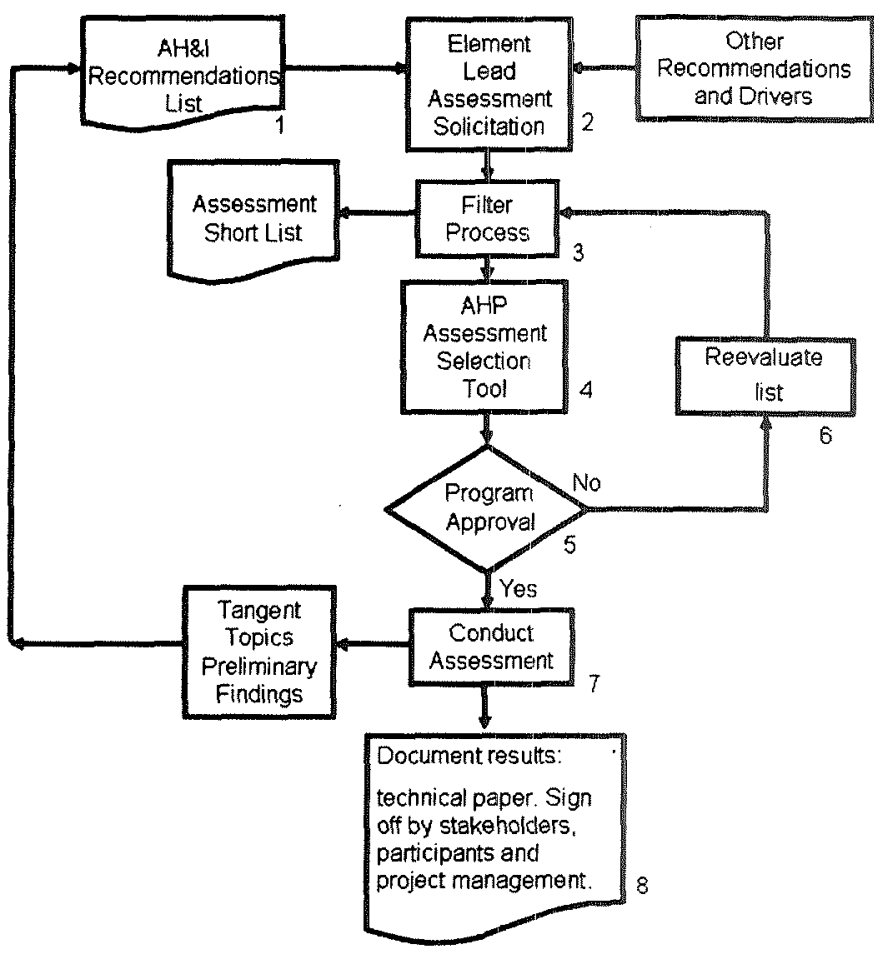

Fig. 2 AH\&l Assessment Selection Process

\section{Integrated Project Team Selection}

Team selection is a central step in the Element Lead Assessment Solicitation subprocess. Individual members of the IPT are selected as representatives of participating organizations. A single point of contact should be the representative of all similar subject expertise, except for those instances where relevant differences are to be considered by the IPT. AH\&I prefers to limit the IPT size to an odd number $(3,5,7)$ for greater functionality.

The human system development team frequently resourced by the AH\&l organization includes representatives from:

- Constellation Program office:

\section{- Advanced Program Office \\ - Advanced Extravehicular Activities (AEVA)}

- Space Life Sciences Directorate, in particular:

- Habitability and Human Factors Division

- Human Adaptation and Countermeasures Division

- Crew and Thermal Systems Division, in particular: 
- Exploration Life Support (ELS)

- Life Support \& Habitability Systems Branch

- EVA and Space Suits System Branch

Participants from each organization represent the technical breadth of the project. Participation is based upon funding allocation and organizational need.

\section{Filter Process}

The filter process is included within the expanded selection process to refine and reduce the assessment topics taken to the AHP prioritization scheme.

The filtering process developed for this application is designed to perform the following:

- Segregate short or long term studies. Short term assessment topics studies are those that can be completed in four to six weeks

- Ensure questionnaire forms are completed and are consistently documented

- Group assessment topics according to common subject matter

- Consolidate duplicate topics or entries

- Identify at least thirteen assessment topics to be scored. If the number of independent assessment topics exceeds thirteen, consider greater consolidation. The maximum assessment candidates chosen should be numerous enough to represent the independent assessment candidates, while minimized to reduce the burden of the prioritization subprocess.

- Preprocess the IPT's suggestions into a short list for preliminary approval and discussion.

A detailed outline of the AH\&I Filter subprocess is provided in Figure 3.

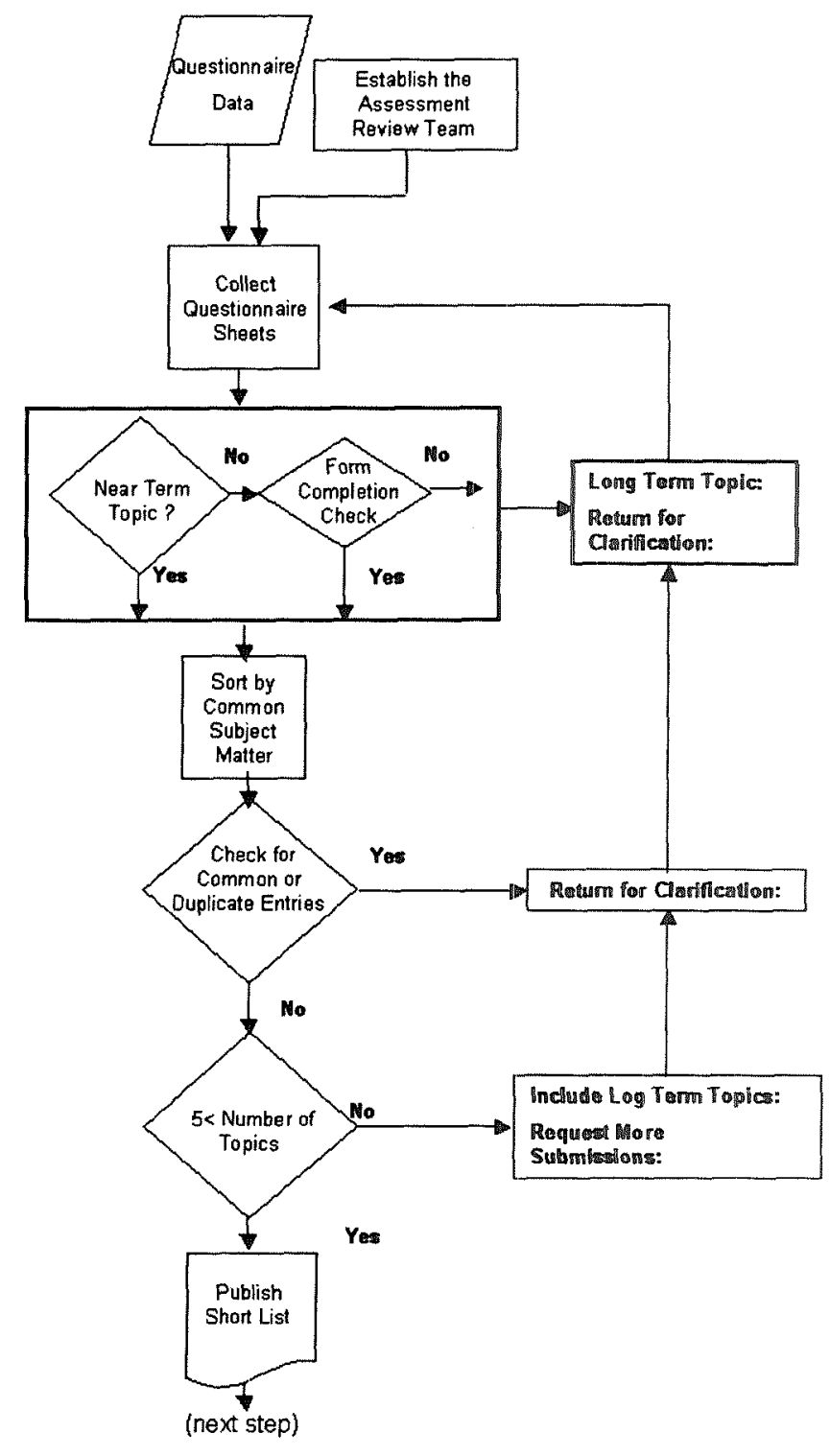

Fig. 3 AH\&I Example Assessment Filter Subprocess

\section{AHP Assessment Selection Tool}

The Analytical Hierarchy Process (AHP) was used to determine the most beneficial assessment topics. The popularity of AHP method and rigor of implementation promotes consistency amongst the comparisons made by the selection team. Specific knowledge of AHP is not required for participation in the comparisons.

The AHP allows for a comparison model to incorporate a hierarchical framework This flexibility allows complex comparisons to be evaluated (Finnie et al, 1999). The flexibility comes at the cost of additional pair-wise comparisons. 
The AHP has been formalized as a two phased process, where the structure of the objectives or goals are defined and the second phase is described as a dialog or discussion where options or choices are made that best satisfy the objectives.

The AH\&I AHP Selection subprocess is defined as the four steps below:

1. Identify the pertinent factors or goals that dictate the usefulness of each assessment topic relative to the IPT's direction. The usefulness of each assessment topics will be graded against all other topics relative to these goals. The structure of these goals may be complex or nested. The AHP typically uses a 1-9 based scale for the pair-wise comparisons within the AHP. Ishizaka et al, 2005 demonstrates the sensitivity of the AHP 1-9 based scale relative to a "compromise" alternative.

2. Survey or question the participant group that is responsible for the evaluation. Pair-wise comparisons are to be performed by a subset of the management team. The management team was chosen to perform the individual pair-wise comparisons, for this particular example. The authors strongly suggest the appropriate responsible parties be required to perform the AHP scoring. This provides a separation of responsibility between the scientific/engineering community and the management community.

3. Evaluate the consistency of the pair-wise comparisons. A valuable feature of the AHP method is a consistency measure, called the consistency ratio (CR). The ratio indicates the consistency of the pair-wise comparisons. Saaty suggests that a CR of more than 0.1 indicates an inconsistent comparison. Such inconsistencies may then be flagged for resolution with the management subgroup.

4. Evaluate the relative weighting of each goal and report. Averages of the individual comparisons are taken and the final recommendations are reported as a consensus of the scoring community. Seldom have we seen a significant spread among the top five assessment topics chosen by the selection team.

Figure 4 is a pictorial representation of a process that satisfies the steps provided above.

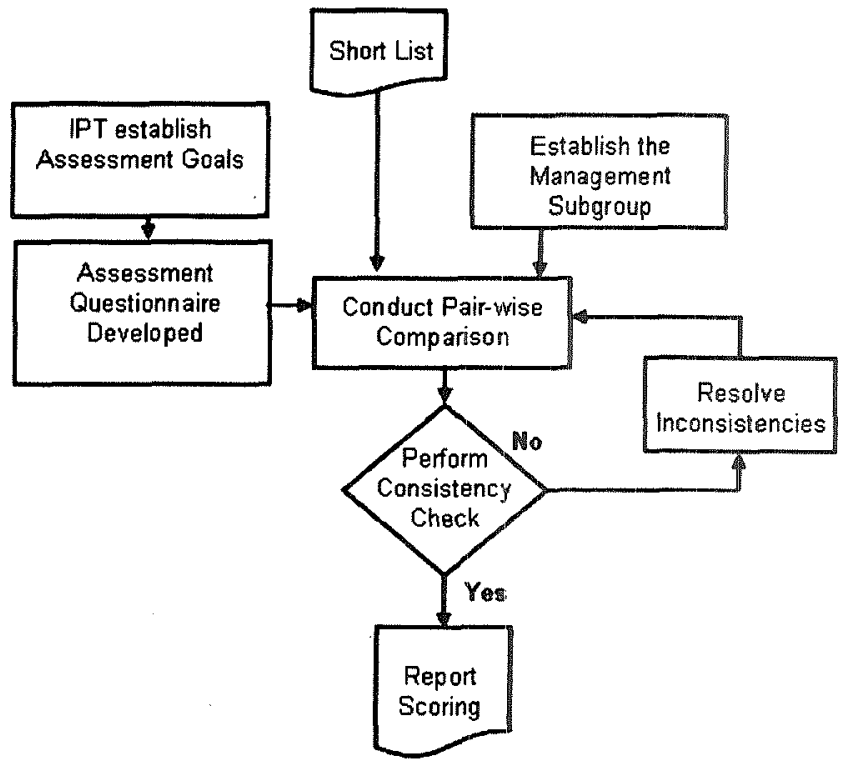

Fig. 4 AH\&I Example AHP Assessment Selection Subprocess

The AH\&I IPT has developed questionnaires to perform the necessary pair-wise comparisons. Benefit to the project is determined as the benefit of continued study relating to the following objectives:

- Stowage Design and Requirements

- Waste handling and processing

- Food preparation and storage

- Commonality of processes and hardware

- Advanced Environmental Monitoring and Control

- Dust Implications and Studies

- Advanced Extra Vehicular Activities

- Radiation Requirements and Integration

The corresponding objective hierarch is shown in Figure 5. The set of beneficial assessments are compared within each objective relative to a set of criteria to maximize the benefit to habitation systems. 


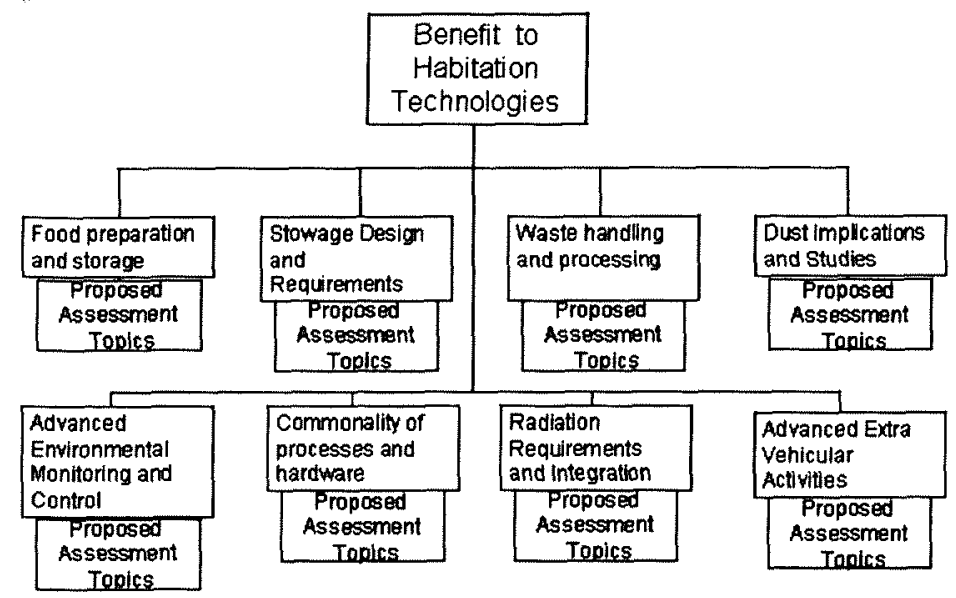

Fig. 5 AH\&I AHP Objective Hierarchy

The benefit of an assessment was determined by a group of seven (Each represented by a Roman numeral) that represented a management point of view. The recommendation of each member is provided in Figure 6.

\begin{tabular}{ll|ll|ll}
\multicolumn{2}{|c|}{ I } & \multicolumn{2}{|c|}{ II } & \multicolumn{2}{c}{ III } \\
Dust & 0.263 & AEVA & 0.222 & Dust & 0.262 \\
AEMC & 0.236 & Rad & 0.216 & Rad & 0.258 \\
AEVA & 0.177 & AEMC & 0.186 & AEVA & 0.163 \\
Rad & 0.135 & Dust & 0.161 & Common & 0.105 \\
\hline & IV & & V & & VI \\
AEVA & 0.191 & Rad & 0.272 & AEVA & 0.165 \\
AEMC & 0.179 & Dust & 0.172 & Rad & 0.159 \\
Common & 0.175 & AEVA & 0.148 & Dust & 0.159 \\
Wasto & 0.123 & Common & 0.122 & AEMC & 0.134 \\
\cline { 1 - 1 } & VII & & & & \\
\hline Rad & 0.248 & & & & \\
AEVA & 0.221 & & & & \\
AEMC & 0.136 & & & & \\
Dust & 0.131 & & & &
\end{tabular}

Fig. 6 AH\&I AHP Individual Results

The average results were computed and provided in Table one.

\author{
Waste \\ 0.068 \\ Stowage $\quad 0.058$ \\ Table 1 AH\&I AHP Average Results
}

The IPT suggested the top five issues for continued study. The IPT was later instructed to investigate subtopics within the four areas identified. Subsequent work in commonality and AEVA has been performed by AH\&I IPT.

\section{CONCLUSION}

Investment in the development of systems engineering practices and tools will continue. These efforts will always be deemed beneficial as management relies on such activities to monitor key decisions and inspect the critical details. Such investments are always intended to promote uniformity, minimize additional expenditure, and maximize benefit.

A generic selection tool has been developed that has aided the engineering community in the development of a repeatable selection methodology. This tool was modified and applied to the selection of cross-cutting issues for study by the NASA's life support and habitation community. Issues identified for future study, by the life support and habitation community, have been pursued as studies or tests.

While the AH\&l team is satisfied with the progress made, some topics for improvement include minimization of an independent set of assessment topics, and the appropriate balance of management and technical comparison factors. The evaluation of many assessment topic yields an impractical number of pair-wise comparisons. While the total number of assessment topics must be minimized, each of the remaining topics must represent a unique assessment option. A stringent filter subprocess can be used to force greater variability between the selection points and minimize the total number of assessment topics. The selection process attempted to maximize management's participation in the selection process; after all, management will reject results they do not approve of. As a result, management was asked to participate in the roles of primary goal definers, pair-wise evaluators, and final approvers. The development of the secondary goals, and formulation of the assessment topics were addressed by the nonmanagement IPT members. We believe this division of labor achieved a balance between the distinct communities.

$\begin{array}{cc}\text { Results } & \text { Average } \\ \text { Rad. } & 0.195 \\ \text { AEVA } & 0.184 \\ \text { Dust } & 0.176 \\ \text { AEMC } & 0.152 \\ \text { Common } & 0.097 \\ \text { Food } & 0.070\end{array}$




\section{ACKNOWLEDGMENTS}

The authors would like to thank the following for their considerable investment into the AH\&l Assessment Selection Process:

Molly Anderson, NASA Johnson Space Center

Bruce Duffield, Jacobs Sverdrup Engineering

Ricki Marshall, Jacobs Sverdrup Engineering

\section{REFERENCES}

1. Finnie, G.R. and Witting, G.E. "An Intelligent Web Tool for Collection of Comparative Survey Data with an Application to IS Curriculum Design", School of Information Technology, Bond University, 1999

2. Ishizaka Alessio, Balkenborg Dieter, Kaplan Todd, "AHP does not like compromises: the role of measurement scales", Joint Workshop on Decision Support Systems, experimental Economics \& eParticipation, Graz,p. 4F5-54, 2005

3. Jones, Harry, "Project Selection for NASA's R\&D Programs" 35th International Conference on Environmental Systems, 11-14 Jul. [2005].

4. Saaty, T.L. "The Analytical Hierarchy Process", Mac Gray-Hill, New York. 1980 